Mathematical Sciences And Applications E-Notes

Volume 2 No. 2 PP. 1-9 (2014) @ MSAEN

\title{
BURSTS/CT-BURSTS DETECTED BY b-SBEC CODES AND SOLID BURSTS UNDETECTED BY CYCLIC CODES
}

\author{
PANKAJ KUMAR DAS
}

(Communicated by İrfan ŞİAP)

\begin{abstract}
In coding theory, several kinds of errors which depend on the nature of the communication channel are to be dealt with and accordingly codes are constructed to detect and correct such errors. Burst, CT-burst and solid burst errors are common in many communication channels. Different types of linear codes have been developed to deal with such errors. It is also quite possible that in communication more than one type of error occur simultaneously. The code which is developed to deal with one type of error may or may not be able to deal with the other type of error.

The paper presents the minimum number of burst/CT-burst errors that will be detected by a $b$-SBEC code. A lower bound on parity check digits for such code is obtained. Further, the paper presents the number of solid burst errors that will be gone detected/undetected by a cyclic code. By a $b$-SBEC code, we mean a linear code that corrects all solid bursts of length $b$ or less.
\end{abstract}

\section{INTRODUCTION AND PRELIMINARIES}

In coding theory, one important study is to investigate the existence of codes that detect and correct errors. Different types of codes have been developed to deal with different types of errors. It was all started with the development of Hamming codes for single errors, then Golay codes for double and triple random errors and thereafter $\mathrm{BCH}$ codes for multiple error correction and many others. Their applications are found in numerous areas of practical interest. These famous codes are developed keeping in mind about random errors. Later it was found that in many communication channels, like radio channels, telephone line, errors do not occur independently but in a clustered way. These errors are known as burst errors. A burst of length $b$, due to Fire [14], may be defined as follows:

Definition 1.1. A burst of length $b$ is a vector whose only non-zero components are among some $b$ consecutive components, the first and the last of which is non-zero.

Extending the work of Hamming [15], Abramson [1] developed codes that correct single and double adjacent errors. The work due to Fire [14] depicted the general

Date: Received: January 1, 2013; Accepted: June 22, 2013.

2010 Mathematics Subject Classification. 94B05, 94B15, $94 \mathrm{~B} 65$.

Key words and phrases. Parity check digit, burst, CT-burst, solid burst, cyclic codes. 
concept (Definition 1.1) of burst errors. Stone [27], and Bridwell and Wolf [6] considered multiple bursts.

Then Chien and Tang [7] observed that in several channels errors do occur in the form of a burst, but not near the end of the vector. Channels due to Alexander, Gryb and Nast [3] fall in this category. Chien and Tang proposed a modification in the definition of a burst, now known as CT-burst, as follows:

Definition 1.2. A CT-burst of length $b$ is a vector whose only non-zero components are confined to some $b$ consecutive positions, the first of which is non-zero.

If in a burst of length $b$ (see Definition 1.1), all the $b$ components in which the non-zero components are confined are non-zero i.e., all the digits among the $b$ components are in error, such type of bursts are known as solid bursts. Such bursts are prevalent in channels viz. semiconductor memory data [17], supercomputer storage system [2]. A solid burst may be defined as follows:

Definition 1.3. A solid burst of length $b$ is a vector with non-zero entries in some $b$ consecutive positions and zero elsewhere.

For more study on burst/CT-burst/solid burst, one may refer to [4, 5, 8, 9, 10 , $13,21,22,23,24,26,28]$ etc.

In [8], the author studied the codes that detect and correct solid bursts of length $b$ or less. We may define such codes as follows:

Definition 1.4. A $b$-Solid Burst Error Correcting ( $b$-SBEC) $(n, k)$ code is a linear code that corrects all solid bursts of length $b$ or less.

In [11], a different type of errors is considered. It was found that in very busy communication channels, solid burst errors repeat themselves. Such errors are named as 2-repeated solid burst of length $b$. The errors are defined as follows:

Definition 1.5. A 2-repeated solid burst of length $b$ is a vector of length $n$ whose only non-zero components are confined consecutively to two distinct sets of $b$ consecutive components.

Theorem 1.1. [11] Any $(n, k)$ linear code over $G F(q)$ that detects any 2-repeated solid burst of length $b$ or less must have at least $2 \log _{q}\left\{\sum_{i=0}^{b}(q-1)^{i}\right\}$ parity check digits.

A generalization of 2-repeated solid burst of length $b$ is considered in paper [12] due to the fact that solid burst errors may repeat themselves more frequently. Such errors are termed as $m$-repeated solid burst of length $b$ and are defined as follows:

Definition 1.6. An $m$-repeated solid burst of length $b$ is a vector of length $n$ whose only non-zero components are confined consecutively to some $m$ distinct sets of $b$ consecutive components.

An elegant class of linear codes, cyclic codes were first introduced by Prange [19] in 1957 as a result of the search for better error correcting codes. They have extremely concise specification and can be efficiently implemented using simple shift registers. Cyclic codes are very useful to detect burst errors. They are also efficient in finding the number of burst errors that goes undetected. Many researchers have studied works in this direction for different types of errors (refer 
e.g., $[16,25])$. In this regard, this paper also presents a study on solid bursts that go detected/undetected by a cyclic code. Further, it obtains the minimum number of bursts/CT-bursts that are detected by a $b$-SBEC code. The paper is organized as follows:

Section 1 i.e., the Introduction gives basic definitions and brief view of the importance of the study of the paper. Section 2 gives the number of bursts/CT-bursts that can be detected by a $b$-SBEC $(n, k)$ code. Minimum number of parity check digits for such a $b$-SBEC code is also obtained. Section 3 presents the number of solid bursts that are detected/undetected by an $(n, k)$ cyclic code. Section 4 is the conclusion.

\section{Detection of Bursts/CT-Bursts by a $b$-SBEC COde}

This section provides two results. One will give the minimum number of detected errors that are in the form of bursts/CT-bursts by a $b$-SBEC $(n, k)$ code. The other will give a lower bound on parity check digits for such codes.

Theorem 2.1. The number of bursts(/CT-bursts) that can be detected by a b-SBEC $(n, k)$ code over $G F(q)(n \geq 2 b)$ is at least

$$
\begin{aligned}
& \sum_{l=1}^{n-2 b+1}\left[(q-1)\left\{1+(n-b-l+1) \sum_{i=1}^{b}(q-1)^{i}+\sum_{i=1}^{b-1}(b-i)(q-1)^{i}\right\}\right. \\
& \left.+\sum_{t=2}^{b}(q-1)^{t}\left\{(q-1)^{b}+(n-b-t-l+1) \sum_{i=1}^{b}(q-1)^{i}+\sum_{i=1}^{b-1}(b-i)(q-1)^{i}\right\}\right] \\
& +(q-1)\left\{(b-1)+\frac{b(b-1)}{2} \sum_{i=1}^{b}(q-1)^{i}+(b-1) \sum_{i=1}^{b-1}(b-i)(q-1)^{i}\right\} \\
& +\sum_{p=2}^{b-2}\left[( q - 1 ) ^ { p } \left\{(b-p)(q-1)^{b}+\frac{(b-p)(b-p-1)}{2} \sum_{i=1}^{b}(q-1)^{i}\right.\right. \\
& \left.\left.+(b-p) \sum_{i=1}^{b-1}(b-i)(q-1)^{i}+\sum_{l=2}^{p} \sum_{i=1}^{b-l}(b-l+1-i)(q-1)^{i}\right\}\right] \\
& +q(q-1)^{b-1} \sum_{l=2}^{b-1} \sum_{i=1}^{b-l}(b-l+1-i)(q-1)^{i} \\
& +(q-1)^{2 b-1}+\sum_{l=2}^{b-1}(q-1)\left\{1+\sum_{i=1}^{l}(l+1-i)(q-1)^{i}\right\} \\
& +\sum_{p=1}^{b-3}\left\{\sum_{l=1}^{p}\left[(q-1)^{b-p-1} \sum_{i=1}^{l}(l+1-i)(q-1)^{i}\right]\right\} .
\end{aligned}
$$

Proof. Let $X$ be the set of all vectors (bursts/CT-bursts) such that all non-zero components of such vectors are confined consecutively in some two distinct sets of $b$ or less consecutive components. Each element of $X$ can always be expressible as the sum (difference) of two solid bursts of length $b$ or less. As a $b$-SBEC $(n, k)$ code corrects all solid bursts of length $b$ or less, such bursts must be in different cosets. As a result, their sum (difference) can not be a codeword. Therefore, the code can 
detect all elements (bursts/CT-bursts) of $X$.

We now calculate the number of such bursts i.e., elements of $X$.

If the first non-zero component of such bursts starts from the first position, then number of such vectors is

$$
\begin{aligned}
& (q-1)\left\{1+(n-b) \sum_{i=1}^{b}(q-1)^{i}+\sum_{i=1}^{b-1}(b-i)(q-1)^{i}\right\} \\
& +(q-1)^{2}\left\{(q-1)^{b}+(n-b-2) \sum_{i=1}^{b}(q-1)^{i}+\sum_{i=1}^{b-1}(b-i)(q-1)^{i}\right\} \\
& +(q-1)^{3}\left\{(q-1)^{b}+(n-b-3) \sum_{i=1}^{b}(q-1)^{i}+\sum_{i=1}^{b-1}(b-i)(q-1)^{i}\right\} \\
& +\ldots \ldots \ldots . . . \\
& +\ldots \ldots \ldots . . . \\
& +(q-1)^{b}\left\{(q-1)^{b}+(n-b-b) \sum_{i=1}^{b}(q-1)^{i}+\sum_{i=1}^{b-1}(b-i)(q-1)^{i}\right\}
\end{aligned}
$$

which can be written as

$$
\begin{aligned}
& (q-1)\left\{1+(n-b) \sum_{i=1}^{b}(q-1)^{i}+\sum_{i=1}^{b-1}(b-i)(q-1)^{i}\right\} \\
& +\sum_{t=2}^{b}\left[(q-1)^{t}\left\{(q-1)^{b}+(n-b-t) \sum_{i=1}^{b}(q-1)^{i}+\sum_{i=1}^{b-1}(b-i)(q-1)^{i}\right\}\right] .
\end{aligned}
$$

If the first non-zero component of such bursts starts from the second position, then number of such vectors can be obtained from expression (2.1) by replacing $n$ by $n-1$. In the same way, we can obtain the number of such bursts of which the starting non-zero position is from $3^{r d}$ position to $(n-2 b+1)^{t h}$ position by replacing $n$ in expression $(2.1)$ by $n-2, \ldots, 2 b$. In general, if the starting non zero position of the bursts is the $l^{\text {th }}$ position $(1 \leq l \leq n-2 b+1)$, the number of such bursts is given by

$$
\begin{aligned}
& (q-1)\left\{1+(n-b-l+1) \sum_{i=1}^{b}(q-1)^{i}+\sum_{i=1}^{b-1}(b-i)(q-1)^{i}\right\} \\
& +\sum_{t=2}^{b}\left[(q-1)^{t}\left\{(q-1)^{b}+(n-b-t-l+1) \sum_{i=1}^{b}(q-1)^{i}+\sum_{i=1}^{b-1}(b-i)(q-1)^{i}\right\}\right] .
\end{aligned}
$$

Thus, the number of such bursts of which the starting non-zero position is from $1^{\text {st }}$ position to $(n-2 b+1)^{t h}$ position is given by

$$
\begin{aligned}
& \sum_{l=1}^{n-2 b+1}\left[(q-1)\left\{1+(n-b-l+1) \sum_{i=1}^{b}(q-1)^{i}+\sum_{i=1}^{b-1}(b-i)(q-1)^{i}\right\}\right. \\
& \left.+\sum_{t=2}^{b}(q-1)^{t}\left\{(q-1)^{b}+(n-b-t-l+1) \sum_{i=1}^{b}(q-1)^{i}+\sum_{i=1}^{b-1}(b-i)(q-1)^{i}\right\}\right] .
\end{aligned}
$$


The number of such bursts of which the starting non-zero position is the $(n-2 b+2)^{t h}$ position is

$$
\begin{aligned}
& (q-1)\left\{1+(b-1) \sum_{i=1}^{b}(q-1)^{i}+\sum_{i=1}^{b-1}(b-i)(q-1)^{i}\right\} \\
& +(q-1)^{2}\left\{(q-1)^{b}+(b-3) \sum_{i=1}^{b}(q-1)^{i}+\sum_{i=1}^{b-1}(b-i)(q-1)^{i}\right\} \\
& +(q-1)^{3}\left\{(q-1)^{b}+(b-4) \sum_{i=1}^{b}(q-1)^{i}+\sum_{i=1}^{b-1}(b-i)(q-1)^{i}\right\} \\
& +\ldots \ldots \ldots . . . \\
& +\ldots \ldots \ldots . . \\
& +(q-1)^{b-2}\left\{(q-1)^{b}+\sum_{i=1}^{b}(q-1)^{i}+\sum_{i=1}^{b-1}(b-i)(q-1)^{i}\right\} \\
& +(q-1)^{b-1}\left\{(q-1)^{b}+\sum_{i=1}^{b-1}(b-i)(q-1)^{i}\right\} \\
& +(q-1)^{b}\left\{\sum_{i=1}^{b-2}(b-1-i)(q-1)^{i}\right\} .
\end{aligned}
$$

The number of such bursts of which the starting non-zero position is the $(n-2 b+3)^{t h}$ position is

$$
\begin{aligned}
& (q-1)\left\{1+(b-2) \sum_{i=1}^{b}(q-1)^{i}+\sum_{i=1}^{b-1}(b-i)(q-1)^{i}\right\} \\
& +(q-1)^{2}\left\{(q-1)^{b}+(b-4) \sum_{i=1}^{b}(q-1)^{i}+\sum_{i=1}^{b-1}(b-i)(q-1)^{i}\right\} \\
& +(q-1)^{3}\left\{(q-1)^{b}+(b-5) \sum_{i=1}^{b}(q-1)^{i}+\sum_{i=1}^{b-1}(b-i)(q-1)^{i}\right\} \\
& +\ldots \ldots \ldots . . . \\
& +\ldots \ldots \ldots . . \\
& +(q-1)^{b-2}\left\{(q-1)^{b}+\sum_{i=1}^{b-1}(b-i)(q-1)^{i}\right\} \\
& +(q-1)^{b-1}\left\{\sum_{i=1}^{b-2}(b-1-i)(q-1)^{i}\right\} \\
& +(q-1)^{b}\left\{\sum_{i=1}^{b-3}(b-2-i)(q-1)^{i}\right\} .
\end{aligned}
$$


Continuing the process, the number of such bursts of which the starting non-zero position is the $(n-b)^{t h}$ position is

$$
\begin{aligned}
& (q-1)\left\{1+(q-1)^{b}+\sum_{i=1}^{b-1}(b-i)(q-1)^{i}\right\} \\
& +(q-1)^{2}\left\{\sum_{i=1}^{b-2}(b-1-i)(q-1)^{i}\right\} \\
& +(q-1)^{3}\left\{\sum_{i=1}^{b-3}(b-2-i)(q-1)^{i}\right\} \\
& +\ldots \ldots \ldots . . . \\
& +\ldots \ldots \ldots . . \\
& +(q-1)^{b-2}\left\{\sum_{i=1}^{2}(3-i)(q-1)^{i}\right\} \\
& +(q-1)^{b-1}\left\{\sum_{i=1}^{1}(2-i)(q-1)^{i}\right\}
\end{aligned}
$$

Thus, the total number of such bursts of which the starting non-zero position is between $(n-2 b+2)^{t h}$ position and $(n-b)^{t h}$ position is given by

$$
\begin{aligned}
& (q-1)\left\{(b-1)+\frac{b(b-1)}{2} \sum_{i=1}^{b}(q-1)^{i}+(b-1) \sum_{i=1}^{b-1}(b-i)(q-1)^{i}\right\} \\
& +\sum_{p=2}^{b-2}\left[( q - 1 ) ^ { p } \left\{(b-p)(q-1)^{b}+\frac{(b-p)(b-p-1)}{2} \sum_{i=1}^{b}(q-1)^{i}\right.\right. \\
& \left.\left.+(b-p) \sum_{i=1}^{b-1}(b-i)(q-1)^{i}+\sum_{l=2}^{p} \sum_{i=1}^{b-l}(b-l+1-i)(q-1)^{i}\right\}\right] \\
& +q(q-1)^{b-1} \sum_{l=2}^{b-1} \sum_{i=1}^{b-l}(b-l+1-i)(q-1)^{i}+(q-1)^{2 b-1} .
\end{aligned}
$$

Finally, we can enumerate the number of such bursts of which the starting non-zero position is within the last $b$ positions. This number is given by

$$
\begin{aligned}
& \sum_{l=2}^{b-1}(q-1)\left\{1+\sum_{i=1}^{l}(l+1-i)(q-1)^{i}\right\} \\
& +\sum_{p=1}^{b-3}\left\{\sum_{l=1}^{p}\left[(q-1)^{b-p-1} \sum_{i=1}^{l}(l+1-i)(q-1)^{i}\right]\right\} .
\end{aligned}
$$

Therefore, the total number of elements (bursts/CT-bursts) of $X$ is given by

$$
\operatorname{expr} .(2.2)+\operatorname{expr} .(2.3)+\operatorname{expr} .(2.4)
$$

i.e.

$$
\sum_{l=1}^{n-2 b+1}\left[(q-1)\left\{1+(n-b-l+1) \sum_{i=1}^{b}(q-1)^{i}+\sum_{i=1}^{b-1}(b-i)(q-1)^{i}\right\}\right.
$$




$$
\begin{aligned}
& \left.+\sum_{t=2}^{b}(q-1)^{t}\left\{(q-1)^{b}+(n-b-t-l+1) \sum_{i=1}^{b}(q-1)^{i}+\sum_{i=1}^{b-1}(b-i)(q-1)^{i}\right\}\right] \\
& +(q-1)\left\{(b-1)+\frac{b(b-1)}{2} \sum_{i=1}^{b}(q-1)^{i}+(b-1) \sum_{i=1}^{b-1}(b-i)(q-1)^{i}\right\} \\
& +\sum_{p=2}^{b-2}\left[( q - 1 ) ^ { p } \left\{(b-p)(q-1)^{b}+\frac{(b-p)(b-p-1)}{2} \sum_{i=1}^{b}(q-1)^{i}+(b-p) \sum_{i=1}^{b-1}(b-i)(q-1)^{i}\right.\right. \\
& \left.\left.+\sum_{l=2}^{p} \sum_{i=1}^{b-l}(b-l+1-i)(q-1)^{i}\right\}\right]+q(q-1)^{b-1} \sum_{l=2}^{b-1} \sum_{i=1}^{b-l}(b-l+1-i)(q-1)^{i} \\
& +(q-1)^{2 b-1}+\sum_{l=2}^{b-1}(q-1)\left\{1+\sum_{i=1}^{l}(l+1-i)(q-1)^{i}\right\} \\
& +\sum_{p=1}^{b-3}\left\{\sum_{l=1}^{p}\left[(q-1)^{b-p-1} \sum_{i=1}^{l}(l+1-i)(q-1)^{i}\right]\right\} .
\end{aligned}
$$

In the following, we obtain a bound on parity check digits of any $b$-SBEC code that is analogous to Reiger's bound (refer [20]; also Theorem 4.15, Peterson and Weldon [18]).

Theorem 2.2. The number of parity check digits for a b-SBEC $(n, k)$ code over $G F(q)$ is at least

$$
2 \log _{q}\left\{\sum_{i=0}^{b}(q-1)^{i}\right\} .
$$

Proof. From Theorem 2.1, a $b$-SBEC $(n, k)$ linear code detects all elements of $X$, i.e. all elements of $X$ can not be a code vector. From Definition 1.5, it is clear that the set $X$ consists of all 2-repeated solid bursts of length $b$ or less. Therefore, the $b$-SBEC $(n, k)$ linear code detects all 2 -repeated solid bursts of length $b$ or less. In view of Theorem 1.1, such a code must have at least $2 \log _{q}\left\{\sum_{i=0}^{b}(q-1)^{i}\right\}$ parity check digits.

\section{CYCLIC CODES AND SOlid BURSTS}

It may be noted that cyclic code is well suited for error detection. It is also well known that an $(n, k)$ cyclic can detect any burst of length $n-k$ or less. However, there are several bursts of length greater than $n-k$ which goes undetected (Refer Theorem $8.5 \& 8.6$, Peterson and Weldon [18]). Now based on these theorems, we have the following results on cyclic codes towards the solid burst error detecting capability.

Theorem 3.1. Every $(n, k)$ cyclic code over $G F(q)$ can detect any solid burst of length $n-k$ or less.

Proof is obvious from the Theorem 8.5, Peterson and Weldon [18]. 
Theorem 3.2. The fraction of solid bursts of length $b>n-k$ that can be undetected by an $(n, k)$ cyclic code over $G F(q)$ is

$$
\begin{gathered}
\frac{1}{(q-1)^{b-1}} \quad \text { if } \quad b-1=n-k, \\
\frac{1}{(q-1)^{1+(n-k)}} \quad \text { if } \quad b-1>n-k .
\end{gathered}
$$

Proof. Consider $r(X)$ to be a solid burst of length $b$. Let $g(X)$ be the generator polynomial of the code. Clearly, $\operatorname{deg} g(X)=n-k$. Then $r(X)=X^{i} r_{1}(X)$ for some non negative integer $i$ and $r_{1}(X)$ is some polynomial with degree $b-1$. As $r(X)$ represents a solid burst of length $b$, so all the coefficients are non-zero which may be chosen by $q-1$ ways. Therefore, there are $(q-1)^{b}$ distinct polynomials $r_{1}(X)$.

The error will be undetected iff $r_{1}(X)$ has $g(X)$ as a factor; that is, $r_{1}(X)=$ $g(X) Q(X)$. Since $g(X)$ has degree $n-k$, so $Q(X)$ must have degree $b-1-(n-k)$. If $b-1=n-k$, then $Q(X)$ is a non-zero constant, and there are $q-1$ values it may take. The ratio of undetected solid bursts to the total number of solid bursts is $\frac{(q-1)}{(q-1)^{b}}=\frac{1}{(q-1)^{b-1}}$. If $b-1>n-k, Q(X)$ may have any of the $q-1$ non-zero field elements as its coefficients. Therefore, there are $(q-1)^{b-1-(n-k)}$ choices of $Q(X)$ which give undetected error pattern. The ratio in this case is

$$
\frac{(q-1)^{b-1-(n-k)}}{(q-1)^{b}}=\frac{1}{(q-1)^{1+(n-k)}} .
$$

Hence the theorem.

\section{Conclusion}

The paper deals with the detected burst/CT-burst errors by a $b$-SBEC code, then with the undetected solid burst errors by a cyclic code. These studies may be extended to other type of errors and other type of codes.

\section{REFERENCES}

[1] Abramson, N.M., A class of systematic codes for non-independent errors. IRE Trans. on Information Theory, IT-5 (1959), no. 4, 150-157.

[2] Arlat, J. and Carter, W.C., Implementation and Evaluation of a $(b, k)$-Adjacent Error- Correcting/Detecting Scheme for Supercomputer Systems. IBM Journal of Research and Development 28 (1984), no. 2, 159-169.

[3] Alexander, A.A., Gryb, R.M. and Nast, D.M., Capabilities of the telephone network for data transmission. Bell System Tech. J., 39 (1960), no. 3, 431-476 .

[4] Argyrides, C.A., Reviriego, P., Pradhan, D.K. and Maestro, J.A., Matrix-Based Codes for Adjacent Error Correction. IEEE Transactions on Nuclear Science, 57 (2010), no. 4, 21062111 .

[5] Bossen, D.C., b-Adjacent Error Correction. IBM Journal of Research and Development, 14 (1970), no. 4, 402-408.

[6] Bridwell, J.D. and Wolf, J.K., Burst distance and multiple-burst correction. Bell System Tech. J., 49 (1970), 889-909,

[7] Chien, R.T. and Tang, D.T., On definitions of a burst. IBM Journal of Research and Development, 9 (1965), no. 4, 292-293.

[8] Das, P.K., Codes Detecting and Correcting Solid Burst Errors. Bulletin of Electrical Engineering and Informatics, 1 (2012), no. 3, 225-232. 
[9] Das, P.K., Codes Detecting and Locating Solid Burst Errors. Romanian Journal of Mathematics and Computer Science. 2 (2012), no. 2, 1-10.

[10] Das, P.K., Blockwise Solid Burst Error Correcting Codes. International Journal on Information Theory, 1 (2012), no. 1, 11-17.

[11] Das, P.K., On 2-Repeated Solid Burst Errors, International Journal in Foundations of Computer Science \& Technology, 3 (2013), no. 3, 41-47.

[12] Das, P.K., Codes on m-repeated solid burst errors, TWMS J. App. Eng. Math., 3 (2013), no. $2,142-146$

[13] Etzion, T., Optimal codes for correcting single errors and detecting adjacent errors. IEEE Trans. Inform. Theory, 38 (1992), 1357-1360.

[14] Fire, P., A class of multiple-error-correcting binary codes for non-independent errors, Sylvania Report RSL-E-2, Sylvania Reconnaissance Systems Laboratory, Mountain View, Calif, (1959).

[15] Hamming, R.W., Error-detecting and error-correcting codes. Bell System Technical Journal, 29 (1950), 147-160.

[16] Jain, S., Moderate density open-loop burst error detection for cyclic codes. Tamsui Oxford Journal of Mathematical Science, 18 (2002), no. 1, 17-29.

[17] Jensen, D.W., Block code to efficiently correct adjacent data and/or check bit errors. Patent no: US 6604222 B1. Date of Patent Aug 5(www.google.com/patents/US6604222). (2003).

[18] Peterson, W.W. and Weldon(Jr.), E.J., Error-Correcting Codes. 2nd edition, The MIT Press, Mass, (1972).

[19] Prange, E., Cyclic Error-correcting Codes in Two Symbols. U.S. Air Force Cambridge Research Centre, AFCRC-TN-57-103, Bedford, Massachusetts, (1957).

[20] Reiger, S. H., Codes for the Correction of Clustered Errors, IRE Trans. Inform. Theory, IT-6 (1960), 16-21.

[21] Schillinger, A.G., A class of solid burst error correcting codes. Polytechnic Institute of Brooklyn, N.Y., Research Rept. PIBMRI. 1223-64, April (1964).

[22] Siap, I., Burst error enumeration of $m$-array codes over rings and its applications. Eur. J. Pure Appl. Math., 3 (2010), no. 4, 653-669.

[23] Siap, I., CT burst error weight enumerator of array codes. Albanian J. Math., 2 (2008), no. 3, 171- 178 .

[24] Sharma, B.D. and Dass, B.K., Adjacent error correcting binary perfect codes. J. Cybernetics, 7 (1977), 9-13.

[25] Sharma, B.D. and Rohtagi, B., Moderate-density 2-repeated burst error detecting cyclic codes. International Journal of Emerging trends in Engineering and Development, 4 (2012), no. $2,49-55$.

[26] Shiva, S.G.S. and Sheng, C.L., Multiple solid burst-error-correcting binary codes. IEEE Trans. Inform. Theory, IT-15 (1969), 188-189.

[27] Stone, J.J., Multiple burst error correction. Information and Control, 4 (1961), 324-331.

[28] Temiz, F. and Siap, V., Linear block and array codes correcting repeated CT-burst errors, Albanian Journal of Mathematics, 7 (2013), no. 2, 77-92.

Department of Mathematics, Shivaji College (University of Delhi), Raja Garden, New Delhi - 110 027, India

E-mail address: pankaj4thapril@yahoo.co.in 